\title{
Dual Tasking and Working Memory in Alcoholism: Relation to Frontocerebellar Circuitry
}

\author{
Sandra Chanraud ',2, Anne-Lise Pitel', Torsten Rohlfing', Adolf Pfefferbaum ${ }^{1,2}$ and Edith V Sullivan*,1 \\ 'Department of Psychiatry and Behavioral Sciences, Stanford University School of Medicine, Stanford, CA, USA; ${ }^{2}$ Neuroscience Program, SRI \\ International, Menlo Park, CA, USA
}

\begin{abstract}
Controversy exists regarding the role of cerebellar systems in cognition and whether working memory compromise commonly marking alcoholism can be explained by compromise of nodes of corticocerebellar circuitry. We tested 17 alcoholics and 31 age-matched controls with dual-task, working memory paradigms. Interference tasks competed with verbal and spatial working memory tasks using low (three item) or high (six item) memory loads. Participants also underwent structural MRI to obtain volumes of nodes of the frontocerebellar system. On the verbal working memory task, both groups performed equally. On the spatial working memory with the high-load task, the alcoholic group was disproportionately more affected by the arithmetic distractor than were controls. In alcoholics, volumes of the left thalamus and left cerebellar Crus I volumes were more robust predictors of performance in the spatial working memory task with the arithmetic distractor than the left frontal superior cortex. In controls, volumes of the right middle frontal gyrus and right cerebellar Crus I were independent predictors over the left cerebellar Crus I, left thalamus, right superior parietal cortex, or left middle frontal gyrus of spatial working memory performance with tracking interference. The brain-behavior correlations suggest that alcoholics and controls relied on the integrity of certain nodes of corticocerebellar systems to perform these verbal and spatial working memory tasks, but that the specific pattern of relationships differed by group. The resulting brain structure-function patterns provide correlational support that components of this corticocerebellar system not typically related to normal performance in dual-task conditions may be available to augment otherwise dampened performance by alcoholics.

Neuropsychopharmacology (2010) 35, 1868-1878; doi: 10.1038/npp.2010.56; published online 21 April 2010
\end{abstract}

Keywords: brain; alcoholism; MRI; cerebellum; frontal cortex; working memory

\section{INTRODUCTION}

Alcoholism has widespread effects on brain structure involving multiple systems and the selective cognitive functions they subserve. Because of the widespread damage sustained, it has been argued that alcoholism's effect on the brain is diffuse and nonselective (Leber and Parsons, 1982; Parsons, 1994; Tivis et al, 1995). This depiction did not consider the alternative possibility that alcoholism affects selective circuitry spanning the anterior to posterior extent of the cerebrum that may make alcoholism's effect on the brain appear nonspecific. Current evidence supports the neural circuitry position and indicates that specific patterns of cerebral alterations (Chanraud et al, 2007; Harris et al, 2008; Jernigan et al, 1991; Pfefferbaum et al, 1997; Sullivan et al, 2000a) and activations (Chanraud-Guillermo et al, 2009; Desmond et al, 2003; Pfefferbaum et al, 2001) characterize alcoholism. Although alcoholism does not typically eliminate a function, injured systems could

*Correspondence: Dr EV Sullivan, Department of Psychiatry and Behavioral Sciences, Stanford University School of Medicine (MC5723), 401 Quarry Road, Stanford, CA 94305-5723, USA, Tel: + 650498 7328, Fax: + 650859 2743, E-mail: edie@stanford.edu Received 6 January 2010; revised 25 March 2010; accepted 27 March 2010 contribute to impaired performance efficiency, especially of executive functions of verbal and spatial working memory, problem solving, and emotional processing that mark alcoholism-related dysfunction (for review see OscarBerman and Marinkovic (2007)).

Classically, impairment in processes comprising executive function, as evidenced by poor inhibitory control, dampened insight, and restricted attentional and working memory capacity, has been attributed to the disruption of prefrontal systems (Fuster, 1999; Smith and Jonides, 1999). In chronic alcoholism, however, alterations in nodes of the corticocerebellar circuitry, in some instances, can be better predictors of executive dysfunction than are alterations in prefrontal regions (Chanraud et al, 2007; Sullivan, 2003). For example, the volume of midbrain white matter, a region common to both corticocerebellar and cerebellocortical fibers, was linked to executive task performance in alcoholdependent individuals (Chanraud et al, 2009). Using diffusion tensor imaging, Chanraud et al showed that the number of white matter fibers linking the midbrain to the pons was smaller in alcohol dependents than nondependent controls and was related to performance in a cognitive flexibility task. Thus, compromise of selective components of corticocerebellar circuitry in alcoholics likely contributes to executive dysfunction affecting impulse control 
(Nixon et al, 2002), conflict processing (De Rosa et al, 2004), and symptoms of disinhibition (Fein and Di Sclafani, 2004; Hada et al, 2000; Noel et al, 2007).

Regarding the working memory impairment, it is relevant to consider the corticocerebellar circuit in light of parallel anatomical findings in nonhuman primates and human alcoholism. In nonhuman primates, two segregated, parallel closed loops within the corticothalamocerebellar circuit have been identified (Kelly and Strick, 2003). One supports executive functions and involves area 46 of the dorsolateral prefrontal cortex, which receives output from cerebellar Crus I and II through the dentate nucleus and thalamus and projects back to the cerebellum through the pons. The other loop supports motor functions and involves the motor cortex, which receives output from lobules IV-VI of the cerebellar cortex through the dentate nucleus and thalamus and projects back through the pons (Kelly and Strick, 2003). In alcoholism, the executive loop is anatomically and functionally altered, and the motor loop while anatomically nearby may be spared (Harper et al, 2003), and therefore potentially available to compensate for the deficits in the executive loop. Functional magnetic resonance imaging (fMRI) studies provide evidence for compensation as well as inefficient processing as they revealed that alcoholics activated either a different neural network from controls (Pfefferbaum et al, 2001) or activated appropriate regions but did so more widely than controls (Desmond et al, 2003) to perform on par with controls.

Here, we sought to examine these separate loops of corticocerebellar circuitry with a dual-task paradigm, which used tasks known from primate studies to recruit this pair of loops. We posited that if the executive loop, known to be compromised in alcoholism, were invoked to process a working memory task, the relatively spared motor loop could be involved to compensate. If, however, the motor loop was already occupied with a task requiring motor control, then it would not be available to augment the function of the executive loop. Consequently, if one loop is already occupied, then its capacity for processing additional material is reduced, thereby curtailing processing efficiency. For this purpose, we used a fully crossed design with verbal and visuospatial working memory tasks, each performed under single-task conditions, under cognitive phonological interference (arithmetic task), and under motor visuospatial interference (perceptuomotor tracking task) (Cocchini et al, 2002; Baddeley and Logie, 1999).

According to neuropsychological dual-task models (King et al, 2007), impaired performance could be, at least partially, attributed to disturbances in bottom-up cerebellothalamocortical loops underlying the regulation of cognitive processes. Because the corticocerebellar circuit is separated into different components (Habas et al, 2009; Krienen and Buckner, 2009), alterations in this circuit could involve either central or domain-specific processing inefficiency. Thus, the dual-task paradigm (Garcia-Villamisar and Della Sala, 2002; Kondo and Osaka, 2004; Oram et al, 2005) could also direct exploration of the question whether alcoholism's functional deficit is global rather than specific and if processing inefficiency could be attributed to compromise of the central executive system.

In controls, we hypothesized that the arithmetic task would interfere with the spatial working memory task and would cause greater interference to verbal working memory as both share domain-specific resources. The perceptuomotor tracking task should interfere only with the spatial working memory task as they share the same domain resources. If global cognitive inefficiency characterizes cognitive processing of alcoholics, dual-task conditions should place no greater demand on either the verbal or the spatial memory system, but would affect efficiency of the global coordination process necessary for performing in a dual-task condition. Therefore, alcoholics should show equal performance decrements in both working memory tasks, whatever the interference task. However, because it has been shown that alcoholics activate a verbal processing system during a spatial task (Pfefferbaum et al, 2001), we hypothesized that even during a spatial working memory task, a phonological component would interfere as much as occurs during a verbal working memory task, again revealing curtailed reserve and processing inefficiency in alcoholics. Finally, to test the hypothesis that there is a specific underlying neuroanatomy selectively related to each task, we conducted correlational analysis between performance on both working memory tasks and tissue volumes in nodes of the corticocerebellar circuit.

\section{MATERIALS AND METHODS}

\section{Participants}

The two groups included 17 alcohol-dependent subjects and 31 healthy controls (Table 1). The alcoholics were recruited from community treatment centers, outpatient clinics, hospitals, and by word of mouth. Controls were recruited through flyers, announcements, or word of mouth. All participants provided written informed consent to participate in studies assessing the impact of alcohol on brain structure and function and received a modest stipend for study participation.

All participants were administered the Structured Clinical Interview for DSM-IV-TR (SCID; First et al, 1998) by a clinical psychologist or research nurse who undergo regular calibration sessions; all diagnostic decisions were made in conference consensus meetings, and ties are broken by a third research clinician. Alcohol dependence was determined by DSM-IV criteria. Exclusion factors were lifetime DSM-IV-TR criteria for other Axis I diagnoses, including schizophrenia, bipolar disorder, attention deficit hyperactivity disorder, and posttraumatic stress disorder; mood disorder other than bipolar was not an exclusion if the depression or anxiety onset postdated the alcoholism onset. One control (rat and ant phobias) and three alcoholics met criteria for anxiety disorder; three alcoholics met criteria for mood (depression) disorder. No control and three alcoholics were taking antidepressants. Drug use or abuse over the last year was not permitted, although two alcohol dependents were found to be infrequent (nonabusing) cannabis users for the last 20 years and their last usage was about 2 weeks before study participation. Lifetime alcohol consumption was estimated and quantified using a modification (Pfefferbaum et al, 1988) of a semi-structured, time-line interview (Skinner, 1982; Skinner and Sheu, 1982). Drinks of each type of alcoholic beverage (wine, beer, spirit) were standardized to units containing approximately $13.6 \mathrm{~g}$ 
Table I Demographic Characteristics of Subjects: Mean (Standard Deviation)

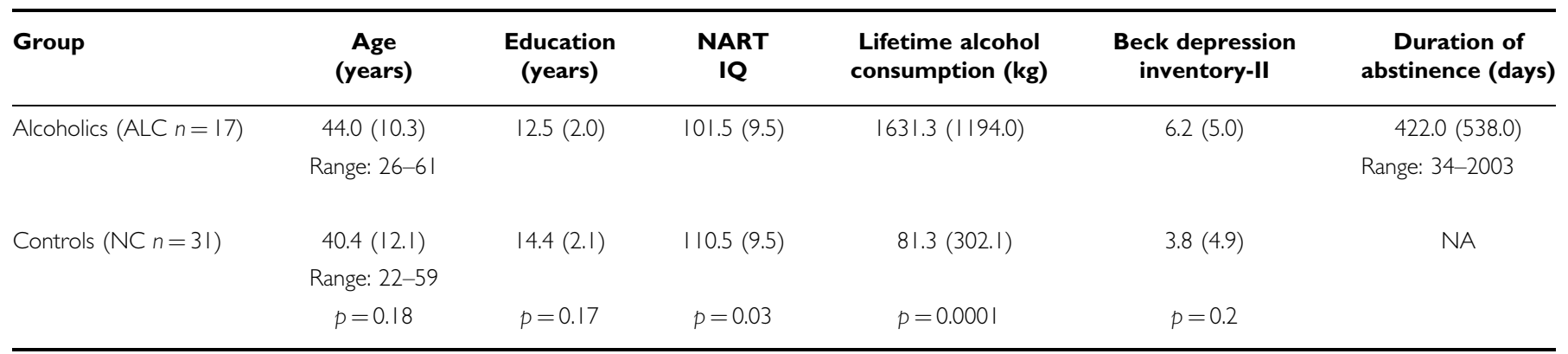

of absolute alcohol and summed over the lifetime. Although toxicology screening was not performed, all subjects completed breathalyzer testing at the beginning of a morning and afternoon testing session. Subjects were excluded if the breathalyzer exceeded 0.0. The diagnostic interview also made inquiries regarding history of medical disorders. Participants with medically uncontrolled hypertension or diabetes were excluded as were those who were positive for HIV infection; because of our ongoing HIV study, all alcoholics and most controls underwent laboratory testing for HIV infection, and none was positive.

Participants also completed cognitive testing and clinical questionnaires. The groups were comparable in age (Table 1), but, mirroring the national prevalence of the sex distribution of alcohol use disorders in the United States (Grant et al, 2003), more women were in the control than the alcoholic group $\left(\chi^{2}(4)=0.16, N=48, p=0.003\right)$. Both groups were of similar level of education $(t(46)=3$, $p=0.17)$ but differed on IQ $(t(43)=-3.031 p=0.03)$ estimated with the National Adult Reading Test (NART; (Nelson, 1982)). The groups did not differ significantly in depressive symptoms detected with the Beck Depression Inventory-II (Beck et al, 1996) $(t(46)=1.72, p=0.094)$.

As expected, alcoholics reported significantly higher lifetime alcohol consumption than the controls $(t$ $(46)=-3.24, p=0.003)$. The average length of lifetime alcohol dependence based on the SCID interview was $20 \pm 10.4$ years, the mean total lifetime alcohol consumption was $1631.3 \pm 1194 \mathrm{~kg}$, and the median duration of abstinence for alcoholics was 82 days (range 34-2003 days). One alcohol-dependent subject had been sober for a long period (2003 days), but all the others had been sober for a shorter time (maximal period $=798$ days).

\section{Tasks}

Verbal and spatial working memory. All conditions followed the same general pattern: presentation of memoranda, imposition of a retention interval, and a period of recall (Figure 1). The specific conditions are described next.

Typically, a memory load must include about six items before interference effects can be observed (Baddeley and Hitch, 1974; Sternberg, 1966). Consequently, we constructed low-load spans of three items as a control condition and high-load spans of six items using verbal or spatial memoranda. The memory task required that the three or six items were recalled in the order of presentation.
Subjects watched and memorized a sequence of visual stimuli presented in different spatial positions in the visual condition and of letters in the verbal condition; after an $8 \mathrm{~s}$ retention interval, subjects tapped out the sequence of spatial positions or of letters on a keypad. Visual stimuli consisted of crosses presented in the right, center, or left part of the screen (in accordance with the nonhuman primate working memory paradigm (Middleton and Strick, 2000)). Letters were always presented in the middle of the screen, and letter sequences could not resemble any words. List items were then presented at a rate of one item every $2 \mathrm{~s}$ ( $1 \mathrm{~s}$ on, $1 \mathrm{~s}$ off). Visual and verbal tasks were balanced across subjects.

Recall performance was based on the percentage of items in a trial successfully recalled in the order presented. For example, if the presented sequence was ' $\mathrm{K} \mathrm{K} \mathrm{L} \mathrm{K} \mathrm{L} \mathrm{J',} \mathrm{and} \mathrm{a}$ participant recalled 'J K K L J J,' the score achieved would be $50 \%$. When participants did not recall the correct number of items within a given trial, one point was deducted for each missing or additional item. For example, if the presented sequence was ' $\mathrm{J} \mathrm{L} \mathrm{J} \mathrm{K} \mathrm{K} \mathrm{L'} \mathrm{and} \mathrm{a} \mathrm{participant} \mathrm{recalled} \mathrm{'} \mathrm{J} \mathrm{L} \mathrm{J} \mathrm{K}$ $\mathrm{K}$,' the score would be $83.33 \%$ (Rudkin et al, 2007). During the delay, participants were asked to retain spatial or verbal information while either waiting without distraction (control condition) or while performing a concurrent task related either to simple arithmetic or to a motor tracking task (interference condition; Figure 1). Both accuracy and reaction time (RT) were recorded.

Interference tasks: arithmetic and tracking. Arithmetic addition involves the phonological loop (Ashcraft and Krause, 2007) whereas spatial tracking taps the visuospatial scratch pad (Baddeley and Logie, 1999). Therefore, we implemented the tasks according to interference effects sought. Participants were instructed to give equal priority to the primary and the interference task. For the arithmetic interference task, participants read three numbers, added them together, and then said whether the sum was less than, equal to, or greater than 15. The percentage of correctly answered problems was recorded. For the tracking interference, participants had to press a key on a computer keyboard corresponding to the position of a target, which was a white circle, presented for $1100 \mathrm{~ms}$ with intervals of $280 \mathrm{~ms}$ during the $8 \mathrm{~s}$ period of retention. Both numbers in the arithmetic task and target positions in the tracking task were randomized. Accuracy and RT were recorded for the primary and interference tasks. 


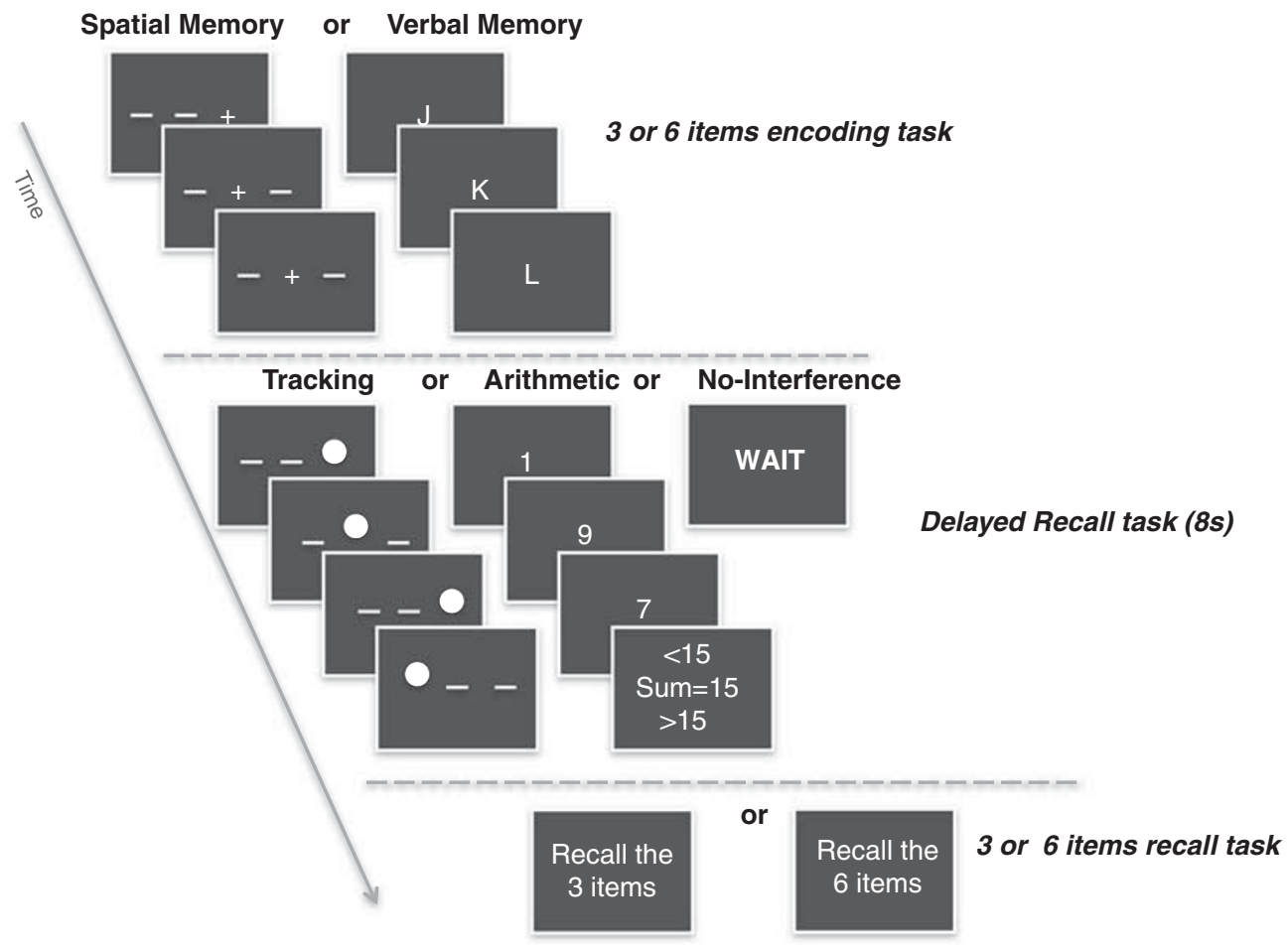

Figure I Dual-tasks paradigm: Subjects watched and memorized a sequence of letters or spatial positions; and after an $8 \mathrm{~s}$ retention interval during which they had to perform either a tracking or an arithmetic task or just wait, subjects tapped out the sequence of letters or of spatial positions on a keypad.

To ensure that the participants were performing the interference task, we set a $60 \%$ level-of-accuracy response criterion for the interference task in each domain for each task (Chan and Newell, 2008). All subjects included reached this level of accuracy during the practice tasks. One subject failed to meet the performance criterion, and thus the data were not included in the analysis.

\section{Cerebral and Cerebellar Tissue Quantification}

Magnetic Resonance Imaging (MRI) acquisition protocol. MRI was conducted on a General Electric 3T clinical system. Structural images for tissue quantification were available for 15 of the 17 alcoholics and 19 of the 31 controls. These data were acquired with a volumetric SPoiled Gradient Recalled (SPGR) sequence $(124,1.25$-mm-thick slices; skip $=0 \mathrm{~mm}$; $\mathrm{TR}=6.5 \mathrm{~ms} ; \mathrm{TE}=1.54 \mathrm{~ms}$; flip angle $=15^{\circ}$; matrix $=256 \times$ 256) for morphometry and dual-echo fast spin-echo (FSE) sequences $(62,2.5$ - $\mathrm{mm}$-thick slices; skip $=0 \mathrm{~mm}$; $\mathrm{TR}=8585 \mathrm{~ms} ; \mathrm{TE}=17 / 102 \mathrm{~ms} ; \quad$ matrix $=256 \times 192)$ for automated fluid-tissue delineation and brain extraction. All images were read by a clinical neuroradiologist to identify space occupying lesions or other dysmorphology indicative of neuropathology that could interfere with morphometric analysis. Additional review of images identified studies with quality too poor for quantification.

MRI quantification. A parcellated template was first created using an average of 24 normal controls spanning the adult age range (Rohlfing et al, 2010). The template was semiautomatically parcellated using an already published description of 116 anatomical brain regions
(Tzourio-Mazoyer et al, 2002). Structural volumes were estimated using the following protocol for each subject: (1) Intensity bias field correction was performed separately on the SPGR and early-echo FSE image. The early-echo FSE bias field was also applied to the late-echo FSE image. (2) The bias-corrected early-echo FSE image was registered to the bias-corrected SPGR image. (3) The bias-corrected SPGR and FSE images were then each passed independently through the FSL Brain Extraction Tool (Smith, 2002) to extract the brain and exclude dura, skull, scalp, and other non-brain tissue. The final brain mask for the SPGR data was then created from the three separate, co-registered channel brain masks using majority voting (ie, a pixel was labeled 'brain' if two out of the three input masks labeled it as such). (4) The brain-extracted SPGR data were registered to the SPGR channel of the SRI24 atlas. (5) All subject images and atlas regions of interest (ROIs) were reformatted to subject coordinate space sampled at $1 \times 1 \times 1 \mathrm{~mm}$ isotropic voxel size. (6) Local tissue for cortical regional statistics were based on a three-compartment segmentation (cerebrospinal fluid (CSF), gray matter, and white matter) map of that subject created with FSL-FAST (Zhang et al, 2001) applied to the brain-extracted SPGR image. Then, gray matter, white matter, and CSF volume of each atlas ROI were determined. For the thalamus and cerebellum, we included the whole local tissue in the analyses because resolution and registration limitations precluded accurate segmentation.

ROIs measured in the corticocerebellar circuit were the superior, middle, and inferior frontal gyri; precentral gyrus; superior parietal gyrus; cerebellar hemispheres and vermian lobules (Schmahmann et al, 2000); and the thalamus (Figure 2). The volumes in each ROI were included in the 


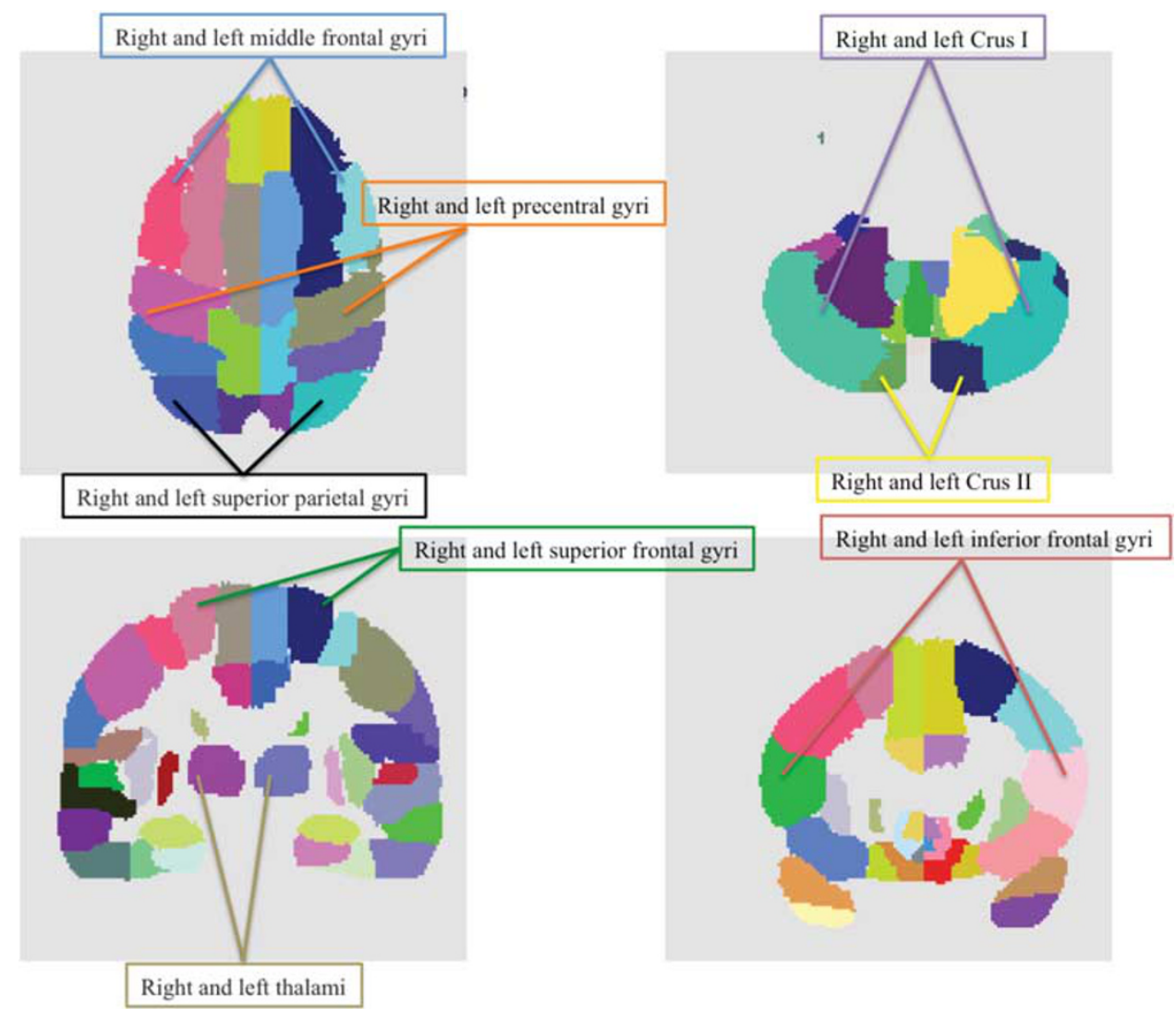

Figure 2 'SRI 24' template parcellation showing the regions of interest investigated in the brain-behavior relationships analysis.

analyses both as raw scores and as $Z$-scores corrected for age and intracranial volume (ICV).

\section{Statistical Analyses}

We first analyzed the effect of the memory load with 'cost' scores, reflecting the cost paid in accuracy through increasing the number of items to memorize without interference. This single-task cost score was calculated as follows: ((three items score-six items score)/three items score $) \times 100$. We also used a cost score to analyze the effects of the interference task, taking into account an individual's baseline performance (accuracy) without interference. The dual-task cost score was calculated as follows: ((single-task score-dual-task score $) /$ single-task score $) \times 100$. All cost scores were converted to age-corrected $Z$-scores based on the performance of the normal control group (Stephens, 2006; Sullivan et $a l, 2000 b)$ and used in all subsequent analyses to remove the effect of normal age.

We conducted two group (controls $v s$ alcoholics) $\times$ two domain (verbal vs spatial) analysis of variance (ANOVA) on the load costs and a two group (controls $v s$ alcoholics) $\times$ two domain (verbal $v s$ spatial) $\times$ two interference condition (arithmetic vs tracking) $\times$ two memory load (3 vs 6) ANOVAs on the dual-task scores. Follow-up $t$-tests identified between-group differences for significant ANOVA effects. RT was subjected to two group $\times$ two memory load $\times$ two domain $\times$ three interference conditions ANOVA and $t$-tests. As groups differed in both NART IQ and percentage of women, the statistical analyses were repeated comparing controls sex and IQ matched to the alcoholics.

Multiple stepwise regression analyses were performed to determine whether brain volumes in nodes of the corticocerebellar circuit predicted performance in either group.

Spearman correlations tested relations between performance and total lifetime alcohol consumption and between performance and duration of abstinence in the alcoholics.

\section{RESULTS}

Table 2 displays the recall accuracy and response time for each group in each condition.

\section{Accuracy}

Effect of memory load: single-task cost. The two-group $\times$ two domain ANOVA testing the change in cost from three to six items in the no-interference condition revealed that neither the group $(\mathrm{F}(1,80)=0.672, p=0.415)$ nor the domain $(F(1,80)=1.213, p=0.274)$ effects were significant, nor was the interaction $(\mathrm{F}(1,80)=3.197, p=0.08)$. Therefore, regardless of item domain, the two groups paid similar costs with the larger memory load in the no-interference (single-task) condition.

Effect of interference: dual-task cost. The two group $\times$ two domain $\times$ two condition (arithmetic or tracking) $\times$ two memory load ANOVA on cost $Z$-scores revealed a significant effect only of domain $(F(1,354)=5.008$; 
$p=0.026)$. The only significant interaction was groupdomain $(\mathrm{F}(1,354)=5.004 ; p=0.026)$, and a tendency of significance was revealed for the four-way interaction involving group $\times$ domain $\times$ number of items $(F(1,354)$ $=3.244 ; p=0.053)$. Post hoc analyses revealed that alcoholics paid a higher cost than controls in the spatial $(t(180)=1.599 ; p=0.041)$ but not the verbal working memory task $(t(180)=-1.277 ; p=0.204)$. In the spatial task, further analyses showed that this effect was due to the arithmetic interference $(t(88)=2.236 ; p=0.028)$ with no effect of the tracking interference $(t(90)=0.180 ; p=0.858)$. In the more challenging six memoranda condition, the group difference was greater than with three memoranda when the distractor was arithmetic $(t \quad(42)=2.592$; $p=0.013)$ and not tracking $(t(43)=-0.612 ; p=0.588)$ (Figure 3).

In general, the same pattern of results was found in the control and alcoholic groups matched on sex and NART IQ.

\section{Reaction Time}

RT differences were assessed with a two group $\times$ two memory load $\times$ two domain $\times$ three interference condition ANOVA that revealed significant effects of number of memoranda $(\mathrm{F}(1,541)=93.701, p<0.001)$ and interference condition $(\mathrm{F}(2,541)=11.755, p<0.001)$, where six items were harder to recall than three items and arithmetic interference attenuated recall more than tracking or no task. Neither the group $(\mathrm{F}(1,541)=0.006, p=0.938)$ nor the domain $(\mathrm{F}(1,541)=1.109, p=0.293)$ effects were significant nor were any interactions (Figure 4 ).

\section{Tracking and Arithmetic Tasks}

We explored whether RT during the tracking task or performance during the arithmetic task differed by group. In no case did the control and alcoholic groups differ significantly.

\section{Group Differences in Regional Brain Volumes}

Between-groups comparisons of $Z$-scores revealed that the alcoholic group had significantly smaller volumes in the right superior frontal $(p=0.019)$ and in the vermian region V1 $(p=0.007)$ than the control group.

\section{Brain-Behavior Correlations}

Using raw scores, no between-groups differences in tissue were present in the ROIs explored, but several predicted brain structure-function correlations were observed. Spatial but not verbal working memory performance correlated with targeted nodes of frontocerebellar circuitry in alcohol dependents and controls. In alcoholics, the dual-task condition eliciting the greatest impairment occurred for the six-position sequence with arithmetic distraction and was the focus of these brain structure-function analyses. Lower performance related to smaller volumes of left cerebellar Crus I $(r=0.53, p=0.045)$, left frontal superior cortex $\quad(r=0.51, \quad p=0.05), \quad$ left thalamus $(r=0.62$, $p=0.014)$, and left precentral region $(r=0.51, p=0.049)$ 

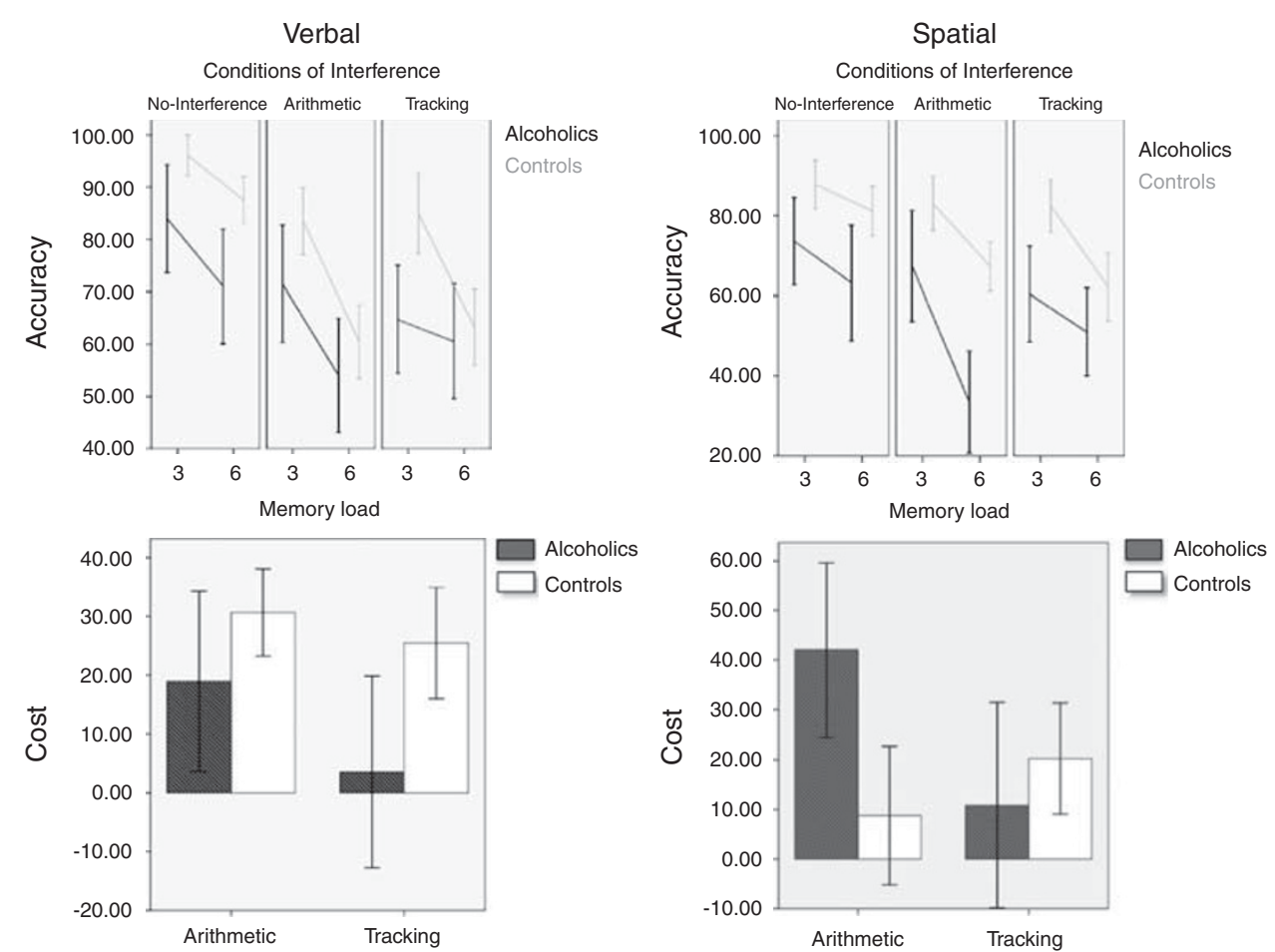

Figure 3 (Top) Mean performance during the verbal working memory task (left) and spatial working memory task (right) for three- and six-item memory load. (Bottom) Mean cost (paid in accuracy through increasing the number of memoranda) during the verbal working memory task (left) and spatial working memory task (right) for six-item memory load.
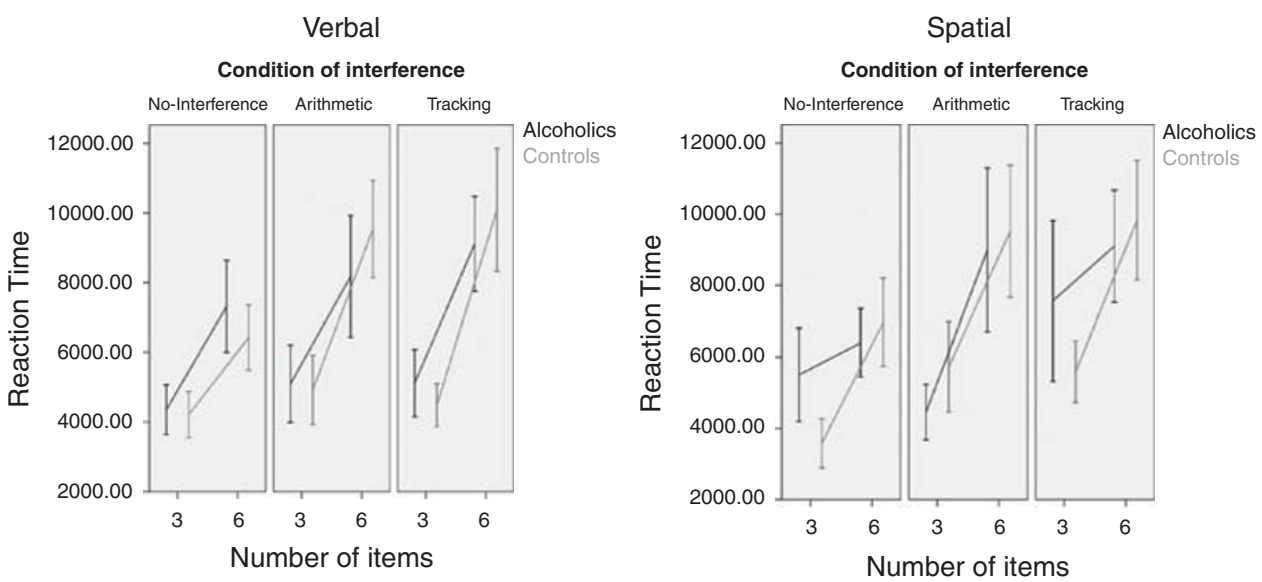

Figure 4 Mean reaction time during the verbal working memory task (left) and spatial working memory task (right) for three- and six-item sequences.

(Figure 5). Multiple regression testing for selectivity of these relations revealed that together these variables accounted for $56 \%$ of the variance with volumes of the left thalamus $(p=0.014)$ and the left cerebellar Crus I $(p=0.05)$ being predictors of performance (compare with $p=0.93$ for left frontal superior cortex). Alcoholics' scores in the sixposition sequence with tracking interference did not correlate with tissue in any node.

In controls, the greatest distraction effect was for the sixposition sequence condition with tracking interference, which correlated with cerebellar Crus I (left $r=0.61$, $p=0.006$; right $r=0.64, p=0.004$ ), middle frontal gyri (left $r=0.52, p=0.024$; right $r=0.63, p=0.005)$, right superior parietal cortex $(r=0.49, p=0.033)$, left thalamus $(r=0.50$, $p=0.03)$, and left precentral region $(r=0.48, p=0.039)$. The three regional volumes with the strongest correlation with performance accounted for $65 \%$ of the variance. Multiple regression with ROIs significant in bivariate correlations identified the right middle frontal gyrus $(p=0.004)$ and the right cerebellar Crus I $(p=0.003)$ as significant independent predictors of performance over the remaining ROIs $(p=0.90$ for left cerebellar Crus I; $p=0.53$ for left thalamus; $p=0.09$ for right superior parietal cortex; and $p=0.55$ for left middle frontal gyrus). Control scores in the six-position sequence with arithmetic interference did not correlate with any node. 

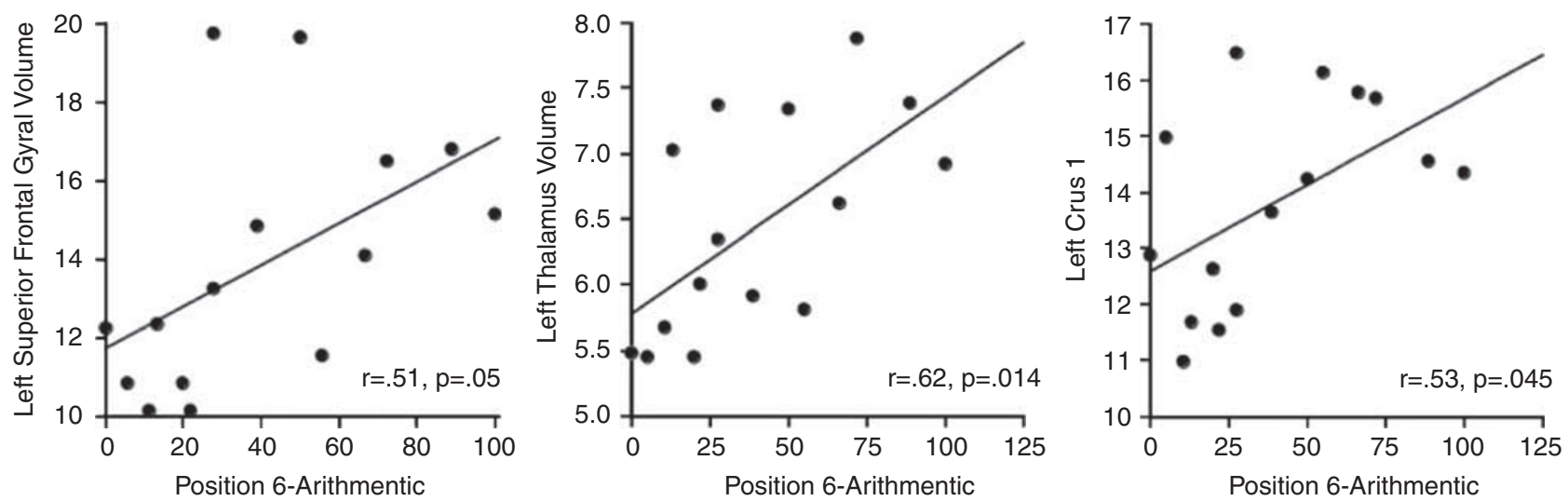

Figure 5 Correlations between the six-item spatial working memory test with the arithmetic task as interference and three regional brain volumes in alcoholics.

Table 3 Patterns of Correlation Between Test Scores and Brain Region Z-Scores

\begin{tabular}{|c|c|c|c|c|c|c|c|c|}
\hline Group & Left precentral & $\begin{array}{l}\text { Left } \\
\text { Crus I }\end{array}$ & $\begin{array}{l}\text { Right } \\
\text { Crus I }\end{array}$ & $\begin{array}{l}\text { Left middle } \\
\text { frontal }\end{array}$ & $\begin{array}{l}\text { Right middle } \\
\text { frontal }\end{array}$ & $\begin{array}{l}\text { Left } \\
\text { thalamus }\end{array}$ & $\begin{array}{l}\text { Left superior } \\
\text { frontal }\end{array}$ & $\begin{array}{c}\text { Right superior } \\
\text { parietal }\end{array}$ \\
\hline Alcoholics (ALC $n=15$ ) & $r=0.45$ & $r=0.26$ & NA & NA & NA & $r=0.58$ & $r=0.52$ & NA \\
\hline \multirow[t]{2}{*}{ Controls (NC $n=19$ ) } & $r=0.45$ & $r=0.4$ & $r=0.42$ & $r=0.68$ & $r=0.44$ & $r=0.41$ & NA & $r=0.19$ \\
\hline & $p=0.05$ & $p=0.09$ & $p=0.07$ & $p<0.001$ & $p=0.5$ & $p=0.08$ & & $p=0.45$ \\
\hline
\end{tabular}

In alcoholics, using $Z$-scores corrected for ICV and age, all the correlations between the scores in the six-item sequence with the arithmetic distraction and brain regions endured. The relation between these test scores and the left precentral regions was attenuated and the one between these test scores and the left cerebellar Crus I was no longer significant (Table 3).

In controls, significant correlations were confirmed between scores in the six-position sequence condition with tracking interference and $Z$-scores for volumes in the bilateral middle frontal gyri and the left precentral region and a strong tendency for significant correlations were revealed between the bilateral cerebellar Crus I and the left thalamus. The significant correlation with the right superior parietal cortex, however, was not confirmed by this analysis.

No other significant correlations were revealed in this analysis.

\section{Correlation Between Alcohol History and Performance}

Only one correlation was significant between alcohol history variables and memory scores; lifetime alcohol consumption correlated with the spatial working memory for the six-item sequence with tracking task as interference $(r=0.62$, $p=0.014)$. Neither other correlations between performance and lifetime alcohol consumption nor any correlations between performance and duration of abstinence were significant.

\section{DISCUSSION}

In this study, alcoholics exhibited performance deficits in spatial but not verbal working memory when engaging in a secondary, cognitive interference. Thus, the alcoholics were marked by selective and not simply global cognitive impairment, possibly attributable to deficits in the central executive system. Further, only the high-demand procedure (high memory load with an interference task) yielded group differences in performance, thus comporting with the concept that costs in cognitive performance arise from exceeding the capacity of a single system resource (Logie et al, 2004). That memory for spatial but not verbal material suffered from retention interval interference from both spatial and verbal tasks in alcoholics but not controls indicates a selective impairment in spatial working memory that is susceptible to distraction regardless of type of material and not a simple unfilled delay (Sullivan et al, 1997). The observed brain-behavior correlations suggest that alcoholics and controls both relied on the integrity of certain nodes of corticocerebellar systems to perform these verbal and spatial working memory tasks but that the specific pattern of relationships differed by group. The resulting pattern in differences provides correlational support that components of this brain system not typically used by controls when dual tasking may be available to augment otherwise dampened performance in alcoholics.

\section{Impact of Interference on Working Memory}

As typically occurs, interference with the simultaneous execution of two separate tasks (Azouvi et al, 1996) impaired working memory processes (Jha et al, 2004; Postle et al, 2005; Sakai et al, 2002), evidenced by reduced accuracy or prolonged RT. Indeed, both memory tasks (letters and positions) that included interfering conditions (arithmetic and tracking) during the working memory 
maintenance period significantly diminished working memory recall and increased the RT in both groups. In controls, interference domains (verbal or spatial) did not differentially affect working memory, whether or not the domain was similar to the modality of the memoranda. Therefore, controls appeared to use different specialized cognitive functions that are involved in visuospatial, verbal, and interference processes and seem to operate largely in parallel. The relative lack of dual-task disruption with particular task combinations has been associated with the operation of a multiple-component working memory system thought to offer online processing and temporary storage of information (Baddeley and Logie, 1999; Baddeley and Hitch, 1986).

Alcoholics showed the same performance decrements as controls when the domain and the conditions had the same modalities (visuospatial working memory and tracking task or verbal working memory and arithmetic task). These results add evidence for a specific deficit in alcoholics that cannot be explained simply by the impact of a general cognitive demand or of limitations in general cognitive capacity. Such a focal deficit leaves open the possibility that unaffected processes remain available to compensate for brain dysfunction. The possibility that alcoholics use compensatory systems to normalize performance, while useful for a single-task situation, can lead to inefficient processing and impairment when two tasks require simultaneous processing or in handling unexpected cognitive challenges and performing multiple concurrent activities (Green et al, 2000). Further, the ability to acquire and perfect new skills with practice and exposure is related to processing capacity (Harvey et al, 2006). The present results indicate that alcohol-dependent subjects have an exacerbated multitasking impairment in spatial working memory processes. For daily activities, alcoholics' impairment should not only be evident when executing two demanding tasks, such as talking on the phone while driving, but also when attempting simple tasks such as memorizing a phone number while looking for a pen. Taken together, these findings confirm and extend previous results suggesting that interference between two tasks ensues as they compete to recruit a common brain system (Klingberg and Roland, 1997).

\section{Brain-Behavior Correlations}

In controls, performance during the dual-task, commonmodality condition, that is, spatial memory with tracking as the interference task, produced the lowest performance and was correlated with volume in the left precentral region, bilateral medial frontal cortex, right superior parietal cortex, left thalamus, and bilaterally in the cerebellar Crus I. Activations in cerebellar Crus I elicited in fMRI studies have been shown during working memory tasks (for reviews see Marvel and Desmond (2010) and Stoodley and Schmahmann (2009)) and continuous finger tracking (Habas and Cabanis, 2008). A role of a frontoparietal network in dual tasking is also consistent with studies supporting its participation in the coordination of concurrent and interfering task processing (Szameitat et al, 2002). More particularly, the median frontal cortex has been associated with the coordinated manipulation of simulta- neously presented information and the involvement of a parietal area in the dual task is in keeping with the hypothesis that a frontoparietal network sustains executive functioning (Salmon and Collette, 2005).

In alcoholics, significant correlations were found only with performance in the most challenging task, that is, spatial working memory with arithmetic as the interference task. Indeed, alcoholics' performance was correlated with left precentral, superior frontal, bilateral thalamus, and left cerebellar Crus I volumes. The sole region correlating with spatial working memory performance in both groups was the left precentral cortex, a region relatively spared in alcoholics as revealed in neuropathology studies (Harper and Corbett, 1990; Harper et al, 2003; Harper and Kril, 1989; Kril et al, 1997). Taken together, the pattern of brain structure-function relations differed by group and may reflect the availability of brain regions as compensatory for the most challenging tasks.

Phonological processing has been shown to impinge on spatial processing resources, thereby reducing processing efficiency of spatial information (Marvel and Desmond, 2010). We, therefore, speculated that both processes would interact more directly in alcoholics than in healthy subjects. The present findings are consistent with the possibility that alcoholics use 'verbal' strategies as compensation to overcome their specific 'spatial' deficit (Pfefferbaum et al, 2001; Tapert et al, 2001). This study also provides association evidence for nodes of lateralized corticocerebellar circuitry to support challenging features of the working memory task in chronic alcoholism. The functional or anatomical derangement of either system in alcoholics supports the selectivity of the cognitive corticocerebellar system as a mechanism contributing to alcoholism-related cognitive impairment and the motor system as having the potential of contributing to compensation of compromised functions. Recent neuroimaging studies on functional connectivity (Habas et al, 2009; Krienen and Buckner, 2009) have confirmed the presence of segregated loops within the corticocerebellar circuit. Although this study was unable to examine such structural segregation, functional imaging studies would have the temporal and spatial resolution to differentiate corticocerebellar loops and challenge their integrity with functional demands.

\section{ACKNOWLEDGEMENTS}

We thank Margaret Rosenbloom, MA, for helpful comments on data acquisition and article, our research assistants (Megan Thompson, Shara Vinco, Oliver Hsu, and Mallika Bhandarkar), research clinicians (Laura Horsch, $\mathrm{PhD}$, Stephanie A Sassoon, $\mathrm{PhD}$, and Karen Jackson, RN) for their work in subject recruitment, clinical evaluation, medical examination, scheduling, screening, and data collection. This work was supported by the National Institute on Alcohol Abuse and Alcoholism (AA010723, AA017168, AA017923, and AA005965) and a Stanford University Dean's Fellowship awarded to Dr Chanraud. All in-house image processing software used herein is available in source code at http://nitrc.org/projects/cmtk/ as part of the Computational Morphomety Toolkit (CMTK), supported by the National Institute of Biomedical Imaging 
and Bioengineering under Grant No. EB008381. The SRI24 atlas is available at http://nitrc.org/projects/sri24/

\section{DISCLOSURE}

None of the authors has any involvement, financial or otherwise, that might potentially bias their work.

\section{REFERENCES}

Ashcraft MH, Krause JA (2007). Working memory, math performance, and math anxiety. Psychon Bull Rev 14: 243-248.

Azouvi P, Jokic C, Van der Linden M, Marlier N, Bussel B (1996). Working memory and supervisory control after severe closedhead injury. A study of dual task performance and random generation. J Clin Exp Neuropsychol 18: 317-337.

Baddeley AD, Hitch GJ (1974). Working memory. In: Bower GH (ed). The Psychology of Learning and Motivation. Academic Press: New York. Vol 8, pp 47-90.

Baddeley AD, Hitch GJ (1986). Working Memory. Oxford University Press: Oxford.

Baddeley AD, Logie RH (1999). Working memory: the multiple component model. In: Shah AMP (ed). Models of Working Memory. Cambridge University Press: New York. pp 28-61.

Beck AT, Steer RA, Brown GK (1996). Manual for the Beck Depression Inventory-II. Psychological Corporation: San Antonio, TX.

Chan JS, Newell FN (2008). Behavioral evidence for task-dependent 'what' versus 'where' processing within and across modalities. Percept Psychophys 70: 36-49.

Chanraud S, Martelli C, Delain F, Kostogianni N, Douaud G, Aubin $\mathrm{HJ}$ et al (2007). Brain morphometry and cognitive performance in detoxified alcohol-dependents with preserved psychosocial functioning. Neuropsychopharmacology 32: 429-438.

Chanraud S, Reynaud M, Wessa M, Penttila J, Kostogianni N, Cachia A et al (2009). Diffusion tensor tractography in mesencephalic bundles: relation to mental flexibility in detoxified alcohol-dependent subject. Neuropsychopharmacology 34: $1223-1232$.

Chanraud-Guillermo S, Andoh J, Martelli C, Artiges E, Aubin HJ, Reynaud $M$ et al (2009). Brain imaging of language reorganization in detoxified alcohol-dependents. Alcohol Clin Exp Res 33: 977-984.

Cocchini G, Logie RH, Della Sala S, MacPherson SE, Baddeley AD (2002). Concurrent performance of two memory tasks: evidence for domain-specific working memory systems. Mem Cognit 30: 1086-1095.

De Rosa E, Desmond JE, Anderson AK, Pfefferbaum A, Sullivan EV (2004). The human basal forebrain integrates old and the new. Neuron 41: 825-837.

Desmond JE, Chen SH, De Rosa E, Pryor MR, Pfefferbaum A, Sullivan EV (2003). Increased fronto-cerebellar activation in alcoholics during verbal working memory: an fMRI study. Neuroimage 19: 1510-1520.

Fein G, Di Sclafani V (2004). Cerebral reserve capacity: implications for alcohol and drug abuse. Alcohol 32: 63-67.

First MB, Spitzer RL, Gibbon M, Williams JBW (1998). Structured Clinical Interview for DSM-IV Axis I Disorders (SCID) Version 2.0. Biometrics Research Department, New York State Psychiatric Institute: New York, NY.

Fuster J (1999). Synopsis of function and dysfunction of the frontal lobe. Acta Psychiatrica Scandinavica 99(S395): 51-57.

Garcia-Villamisar D, Della Sala S (2002). Dual-task performance in adults with autism. Cogn Neuropsychiatry 7: 63-74.

Grant BF, Dawson DA, Stinson FS, Chou PS, Kay W, Pickering R (2003). The Alcohol Use Disorder and Associated Disabilities
Interview Schedule-IV (AUDADIS-IV): reliability of alcohol consumption, tobacco use, family history of depression and psychiatric diagnostic modules in a general population sample. Drug Alcohol Depend 71: 7-16.

Green MF, Kern RS, Braff DL, Mintz J (2000). Neurocognitive deficits and functional outcome in schizophrenia: are we measuring the 'right stuff? Schizophr Bull 26: 119-136.

Habas C, Cabanis EA (2008). Neural correlates of simple unimanual discrete and continuous movements: a functional imaging study at 3 T. Neuroradiology 50: 367-375.

Habas C, Kamdar N, Nguyen D, Prater K, Beckmann CF, Menon V et al (2009). Distinct cerebellar contributions to intrinsic connectivity networks. J Neurosci 29: 8586-8594.

Hada M, Porjesz B, Begleiter H, Polich J (2000). Auditory P3a assessment of male alcoholics. Biol Psychiatry 48: 276-286.

Harper C, Corbett D (1990). Changes in the basal dendrites of cortical pyramidal cells from alcoholic patients - a quantitative Golgi study. J Neurol Neurosurg Psychiatry 53: 856-861.

Harper C, Dixon G, Sheedy D, Garrick T (2003). Neuropathological alterations in alcoholic brains. Studies arising from the New South Wales Tissue Resource Centre. Prog Neuropsychopharmacol Biol Psychiatry 27: 951-961.

Harper C, Kril JJ (1989). Patterns of neuronal loss in the cerebral cortex in chronic alcoholic patients. J Neurol Sci 92: 81-89.

Harris GJ, Jaffin SK, Hodge SM, Kennedy D, Caviness VS, Marinkovic K et al (2008). Frontal white matter and cingulum diffusion tensor imaging deficits in alcoholism. Alcohol Clin Exp Res 32: 1001-1013.

Harvey PD, Reichenberg A, Romero M, Granholm E, Siever LJ (2006). Dual-task information processing in schizotypal personality disorder: evidence of impaired processing capacity. Neuropsychology 20: 453-460.

Jernigan TL, Butters N, DiTraglia G, Schafer K, Smith T, Irwin M et al (1991). Reduced cerebral grey matter observed in alcoholics using magnetic resonance imaging. Alcohol Clin Exp Res 15: 418-427.

Jha AP, Fabian SA, Aguirre GK (2004). The role of prefrontal cortex in resolving distractor interference. Cogn Affect Behav Neurosci 4: 517-527.

Kelly RM, Strick PL (2003). Cerebellar loops with motor cortex and prefrontal cortex of a nonhuman primate. J Neurosci 23: 8432-8444.

King JA, Colla M, Brass M, Heuser I, von Cramon D (2007). Inefficient cognitive control in adult ADHD: evidence from trialby-trial Stroop test and cued task switching performance. Behav Brain Funct 3: 42.

Klingberg T, Roland PE (1997). Interference between two concurrent tasks is associated with activation of overlapping fields in the cortex. Brain Res Cogn Brain Res 6: 1-8.

Kondo H, Osaka N (2004). Susceptibility of spatial and verbal working memory to demands of the central executive. Jpn Psychol Res 46: 86-97.

Krienen FM, Buckner RL (2009). Segregated fronto-cerebellar circuits revealed by intrinsic functional connectivity. Cereb Cortex 19: 2485-2497.

Kril JJ, Halliday GM, Svoboda MD, Cartwright H (1997). The cerebral cortex is damaged in chronic alcoholics. Neuroscience 79: 983-998.

Leber WR, Parsons OA (1982). Premature aging and alcoholism. Int J Addict 17: 61-88.

Logie RH, Cocchini G, Delia Sala S, Baddeley AD (2004). Is there a specific executive capacity for dual task coordination? Evidence from Alzheimer's disease. Neuropsychology 18: 504-513.

Marvel CL, Desmond JE (2010). The contributions of cerebrocerebellar circuitry to executive verbal working memory. Cortex (E-pub ahead of print 9 September 2009).

Middleton FA, Strick PL (2000). Basal ganglia and cerebellar loops: motor and cognitive circuits. Brain Res Brain Res Rev 31: $236-250$ 
Nelson HE (1982). The National Adult Reading Test (NART). Nelson Publishing Company: Windsor, Canada.

Nixon SJ, Tivis R, Ceballos N, Varner JL, Rohrbaugh J (2002). Neurophysiological efficiency in male and female alcoholics. Progress Neuropsychopharmacol Biol Psychiatry 26: 919-927.

Noel X, Van der Linden M, d'Acremont M, Bechara A, Dan B, Hanak C et al (2007). Alcohol cues increase cognitive impulsivity in individuals with alcoholism. Psychopharmacology (Berl) 192: 291-298.

Oram J, Geffen GM, Geffen LB, Kavanagh DJ, McGrath JJ (2005). Executive control of working memory in schizophrenia. Psychiatry Res 135: 81-90.

Oscar-Berman M, Marinkovic K (2007). Alcohol: effects on neurobehavioral functions and the brain. Neuropsychol Rev 17: 239-257.

Parsons OA (1994). Determinants of cognitive deficits in alcoholics: the search continues. Clin Neuropsychol 8: 39-58.

Pfefferbaum A, Desmond JE, Galloway C, Menon V, Glover GH, Sullivan EV (2001). Reorganization of frontal systems used by alcoholics for spatial working memory: an fMRI study. Neuroimage 14: 7-20.

Pfefferbaum A, Rosenbloom MJ, Crusan K, Jernigan TL (1988). Brain CT changes in alcoholics: the effects of age and alcohol consumption. Alcohol Clin Exp Res 12: 81-87.

Pfefferbaum A, Sullivan EV, Mathalon DH, Lim KO (1997). Frontal lobe volume loss observed with magnetic resonance imaging in older chronic alcoholics. Alcohol Clin Exp Res 21: 521-529.

Postle BR, Desposito M, Corkin S (2005). Effects of verbal and nonverbal interference on spatial and object visual working memory. Mem Cognit 33: 203-212.

Rohlfing T, Zahr NM, Sullivan EV, Pfefferbaum A (2010). The SRI24 multichannel atlas of normal adult human brain structure. Hum Brain Mapp (E-pub ahead of print 16 December 2009).

Rudkin SJ, Pearson DG, Logie RH (2007). Executive processes in visual and spatial working memory tasks. Q J Exp Psychol (Colchester) 60: 79-100.

Sakai K, Rowe JB, Passingham RE (2002). Active maintenance in prefrontal area 46 creates distractor-resistant memory. Nat Neurosci 5: 479-484.

Salmon E, Collette F (2005). Functional imaging of executive functions. Acta Neurol Belg 105: 187-196.

Schmahmann JD, Doyon J, Toga AW, Petrides M, Evans AC (2000). MRI Atlas of the Human Cerebellum. Academic Press: San Diego.

Skinner HA (1982). Development and Validation of a Lifetime Alcohol Consumption Assessment Procedure. Addiction Research Foundation: Toronto, Canada.
Skinner HA, Sheu WJ (1982). Reliability of alcohol use indices: the lifetime drinking history and the MAST. J Stud Alcohol 43: $1157-1170$

Smith EE, Jonides J (1999). Storage and executive processes in the frontal lobes. Science 293: 1657-1661.

Smith SM (2002). Fast robust automated brain extraction. Hum Brain Mapp 17: 143-155.

Stephens R (2006). Age-related decline in Digit-Symbol performance: eye-movement and video analysis. Arch Clin Neuropsychol 21: 101-107.

Sternberg S (1966). High-speed scanning in human memory. Science 153: 652-654.

Stoodley CJ, Schmahmann JD (2009). Functional topography in the human cerebellum: a meta-analysis of neuroimaging studies. Neuroimage 44: 489-501.

Sullivan EV (2003). Compromised pontocerebellar and cerebellothalamocortical systems: speculations on their contributions to cognitive and motor impairment in nonamnesic alcoholism. Alcohol Clin Exp Res 27: 1409-1419.

Sullivan EV, Rosenbloom MJ, Lim KO, Pfefferbaum A (2000a). Longitudinal changes in cognition, gait, and balance in abstinent and relapsed alcoholic men: relationships to changes in brain structure. Neuropsychology 14: 178-188.

Sullivan EV, Rosenbloom MJ, Pfefferbaum A (2000b). Pattern of motor and cognitive deficits in detoxified alcoholic men. Alcohol Clin Exp Res 24: 611-621.

Sullivan EV, Shear PK, Zipursky RB, Sagar HJ, Pfefferbaum A (1997). Patterns of content, contextual, and working memory impairment in schizophrenia and nonamnesic alcoholism. Neuropsychology 11: 195-206.

Szameitat AJ, Schubert T, Muller K, Von Cramon DY (2002). Localization of executive functions in dual-task performance with fMRI. J Cogn Neurosci 14: 1184-1199.

Tapert SF, Brown GG, Kindermann SS, Cheung E, Frank LR, Brown SA (2001). fMRI measurement of brain dysfunction in alcohol dependent young women. Alcohol Clin Exp Res 25: 236-245.

Tivis R, Beatty WW, Nixon SJ, Parsons OA (1995). Patterns of cognitive impairment among alcoholics: are there subtypes? Alcohol Clin Exp Res 19: 496-500.

Tzourio-Mazoyer N, Landeau B, Papathanassiou D, Crivello F, Etard O, Delcroix N et al (2002). Automated anatomical labeling of activations in SPM using a macroscopic anatomical parcellation of the MNI MRI single-subject brain. Neuroimage 15: 273-289.

Zhang Y, Brady M, Smith S (2001). Segmentation of brain MR images through a hidden Markov random field model and the expectation maximization algorithm. IEEE Trans Med Imaging 20: 45-57. 